\title{
Radiological Imaging Analysis of Cervical Expansive Open-door Laminoplasty Causing C5 Palsy
}

\author{
Luo Jianxian, Liu Dongming, Chen Tianjun, Zhang Guowei, Yang Hua, Ji Zhisheng*, \\ Lin Hongsheng*
}

Department of Orthopedics, the First Affiliated Hospital of Jinan University, Guangzhou, China

Email address:

tzhishengji@jnu.edu.cn (Ji zhisheng), tlinhsh@jnu.edu.cn (Lin Hongsheng)

${ }^{*}$ Corresponding author

To cite this article:

Luo Jianxian, Liu Dongming, Chen Tianjun, Zhang Guowei, Yang Hua, Ji Zhisheng, Lin Hongsheng. Radiological Imaging Analysis of Cervical Expansive Open-door Laminoplasty Causing C5 Palsy. International Journal of Neurosurgery. Vol. 4, No. 2, 2020 , pp. 46-49. doi: $10.11648 /$ j.ijn.20200402.15

Received: September 2, 2020; Accepted: September 11, 2020; Published: September 19, 2020

\begin{abstract}
The aim of this research is to explore the difference of imaging parameters in patients with C5 palsy after cervical expansive open-door laminoplasty. A retrospective analysis was made on the clinical data of 62 patients who were diagnosed as multiple cervical spondylotic myelopathy and operated with cervical expansive open-door laminoplasty using Centerpiece titanium miniplate fixation. According to weather $\mathrm{C} 5$ nerve root palsy occurred after surgery, the patients were divided into normal group (group A with 8 cases) and paralysis group (group B with 54 cases). The preoperative and postoperative cervical spine angle (C2-7) enlargement rate of spinal canal, distance of spine cord shifting and cervical curvature between two groups were measured. Compare with group A $\left(27.38 \pm 1.99^{\circ}\right)$, the average lamina open angle of group B $\left(34.56 \pm 5.55^{\circ}\right)$ was larger, which were found statistically significant difference $(P<0.05)$. In addition, there were no significant differences between two groups in $\mathrm{C} 3$ or $\mathrm{C} 7$ lamina open angle. However, significant differences were found between two groups in C4, C5 and C6 lamina open angle. The average enlargement rate of spinal canal and distance of spine cord shifting of group B were larger than group A, which was statistically differences $(P<0.05)$. The opening angle of the lamina, the expansion rate of the spinal canal, and the distance of the spinal cord may prevent the occurrence of cervical 5 nerve root palsy in cervical expansive open-door laminoplasty.
\end{abstract}

Keywords: Cervical Open-Door Laminoplasty; Multiple Cervical Spondylotic Myelopathy; C5 Nerve Root Palsy

\section{Introduction}

Cervical spondylotic myelopathy (CSM) is a common neurological disease [1]. Multi-segment cervical spondylotic myelopathy (MCSM) refers to cervical spondylotic myelopathy involving 3 or more than 3 segments [2]. It often occurs with multiple factors, such as spinal stenosis and ossification of the posterior longitudinal ligament [3]. The posterior cervical single-door expansion spinal canal plasty was first proposed by Japanese scholar Hirabayashi [4], and it has been regarded by many scholars as en effective methods for the treatment of MCSM. It is suitable for multi-segment cervical spondylotic myelopathy caused by cervical disc herniation [5], osteophyte formation on the posterior edge of the vertebral body [6], ossification of posterior longitudinal ligament (OPLL) [7], or ossification of ligamentum flavum. The traditional single door opening technique proposed by Hirabayashi is to fix the opened lamina with silk thread to the paravertebral tissues. The incidence of postoperative axial pain and "re-closing" phenomenon is high [8]. Although compared with the traditional surgical method, the new surgical method reduced the postoperative "re-closing" phenomenon, axial pain and C5 nerve. However, the existence of these complications, especially C5 nerve root palsy, is still an event that troubles spinal surgeons and affects patients' satisfaction after surgery [7]. In order to explore the cause of postoperative C5 nerve root palsy, this study evaluated the opening angle of the posterior cervical single-door Centerpiece titanium plate internal fixation, the degree of postoperative spinal cord movement, and the degree of spinal canal expansion. 


\section{Material and Methods}

\subsection{General Information}

Retrospective analysis of patients diagnosed in our hospital during March 2010 March 2018 as multi-segment cervical spondylosis with cervical spine posterior single-door spinal canal augmentation Centerpiece titanium plate fixation. Clinical data with complete follow-up records. A total of 62 samples were collected in strict compliance with inclusion and exclusion criteria. They were divided into normal group (A group) and paralysis group (B group) according to whether C5 nerve root paralysis occurred after operation. Among them, 54 patients (40 males and 14 women, average age of $61.48 \pm 10.62$ years) in A group and eight patients in B group (6 men and 2 women, average age of $58.90 \pm 11.19$ years) were enrolled.

\subsection{Inclusion and Exclusion Criteria}

Inclusion criteria: (1) patients who meet the diagnostic criteria of multi-segment cervical spondylosis; (2) patients with titanium plate fixation Centerpiece single-door spinal canal enlargement in our hospital. Exclusion criteria: (1) patients with cerebrovascular disease, multiple peripheral neuritis, amyotrophic lateral sclerosis, intraspinal tumors, cervical infection, trauma; (2) patients with kyphosis and cervical instability.

\subsection{Method of Operation}

After tracheal intubation and general anesthesia, the patient is placed in the prone position, connected to the electrophysiological monitoring device, and the Mayfield head holder is fixed in the neck slightly flexed position. After the C-arm fluoroscopy locates the surgical site of the patient, we make an incision from the middle of the back of the neck, cut the skin, subcutaneous, and deep fascia along the nape line in turn, and peel it layer by layer to the supraspinous ligament and expose the surgical segment board. During the operation, we try to preserve the muscles and ligaments of the $\mathrm{C} 2$ and $\mathrm{C} 7$ spinous processes. We choose the side with the heavier symptoms as the door opening side, and then use a drill to groove and penetrate the entire lamina at the place where the lamina and lateral mass move. In addition, the contralateral side is ground to the deep cortex of the lamina. Next, we slowly lift the lamina to the hilar axis and separate the adherent ligamentum flavum and dural sac. Finally, we put the Centerpiece titanium plate on the lamina and side block on the side of the door. During the operation, we need to pay attention to adjusting the lamina of the portal axis to prevent the lamina from rupture and retraction.

\subsection{Measurement Indicators}

Single door lamina opening angle: On the CT cross-sectional view of the cervical spine, the innermost points of the facet joints on both sides are connected, that is, the line connecting the anterior and inner side of the articular process and the pedicle, the angle between the inner vertex of the door opening side and the inner vertex of the door shaft $\alpha$ angle) is the door opening angle.

Spinal canal expansion rate $=$ (postoperative spinal canal cross-sectional area-preoperative spinal canal cross-sectional area) / preoperative spinal canal cross-sectional area $\times 100 \%$.

Spinal posterior distance: The distance from the anterior and posterior edges of the spinal cord to the posterior edge of the same level of the vertebral body on the sagittal CT of the cervical spine was measured. The difference between the distance before and after the operation is the distance of the spinal cord [9].

\subsection{Statistical Analysis}

SPSS19.0 statistical software was used to analyze the data. $\mathrm{P}<0.05$ was considered statistically significant.

\section{Results}

The independent sample $\mathrm{t}$ test was used to compare the opening angles of the $\mathrm{C} 3-\mathrm{C} 7$ lamina between the two groups (Figure 1). The average opening angles of the $\mathrm{C} 3-\mathrm{C} 7$ lamina in the normal group (group A) were $25.17 \pm 2.88^{\circ}, 27.98 \pm 3.59^{\circ}$, $27.61 \pm 2.08^{\circ}, 28.02 \pm 2.19^{\circ}$ and $27.58 \pm 0.84^{\circ}$, and the overall average laminar opening angle was $27.38 \pm 1.99^{\circ}$. In the paralysis group (group B), the average opening angles of the lamina of $\mathrm{C} 3-\mathrm{C} 7$ were $28.66 \pm 3.03^{\circ}, 34.13 \pm 6.92^{\circ}, 35.91 \pm 6.28^{\circ}$, $35.71 \pm 6.86^{\circ}$ and $28.06^{\circ}$, and the overall average lamina opening angle was $34.56 \pm 5.55^{\circ}$. After two independent sample t-tests, there was no statistically significant difference in the opening angle of the $\mathrm{C} 3$ and $\mathrm{C} 7$ lamina between the two groups $(\mathrm{P}>0.05)$. However, the difference in the opening angle of each lamina of C4-C6 and the overall average lamina opening angle was statistically significant $(\mathrm{P}<0.05)$. It can be considered that the occurrence of postoperative $\mathrm{C} 5$ nerve root palsy is related to the larger opening angle of the lamina.

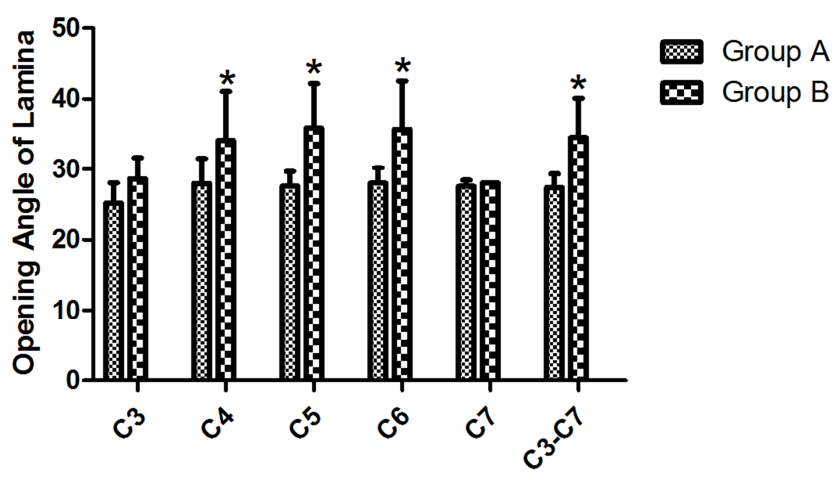

Figure 1. Comparison of the opening angle $\left(^{\circ}\right)$ of each segment of the lamina between the two groups.

In the normal group (group A), the average spinal canal expansion rate of C3-C7 was $37.81 \pm 13.68 \%, 42.32 \pm 12.01 \%$, $44.58 \pm 13.52 \%, 38.89 \pm 14.64 \%$ and $46.09 \pm 10.08 \%$, and the overall average spinal canal expansion rate was $41.68 \pm 9.87 \%$ (Figure 2). The average distance of the spinal cord in each segment of $\mathrm{C} 3-\mathrm{C} 7$ was $0.50 \pm 0.38 \mathrm{~mm}, 0.82 \pm 0.41 \mathrm{~mm}$, $1.07 \pm 0.60 \mathrm{~mm}, 0.92 \pm 0.51 \mathrm{~mm}$, and $0.46 \pm 0.33 \mathrm{~mm}$, and the average distance of the whole spinal cord was $0.76 \pm 0.25 \mathrm{~mm}$. 
In the paralysis group (group B), the average spinal canal expansion rates of C3-C7 were $55.30 \pm 15.23 \%, 56.00 \pm 12.84 \%$, $50.99 \pm 8.39 \%, \quad 55.64 \pm 12.04 \%$, and $69.08 \%$. The overall average spinal canal expansion rate was $54.91 \pm 6.70 \%$. C3-C7 spinal cord posterior distance averaged $1.11 \pm 0.97 \mathrm{~mm}$, $1.48 \pm 0.58 \mathrm{~mm}, 1.73 \pm 0.82 \mathrm{~mm}, 1.68 \pm 0.81 \mathrm{~mm}$, and $0.92 \pm 0.74$ $\mathrm{mm}$, and the overall spinal cord posterior distance averaged $1.38 \pm 0.44 \mathrm{~mm}$. After statistical analysis, the difference between the two groups in the average spinal canal enlargement rate and the average spinal cord retraction distance was statistically significant $(\mathrm{P}<0.05)$ (Figure 3 ). The average spinal canal enlargement rate and the average spinal retraction distance in the paralysis group were larger than those in the normal group. It can be considered that the occurrence of postoperative $\mathrm{C} 5$ nerve root palsy is related to the expansion of the spinal canal and the distance of the spinal cord.

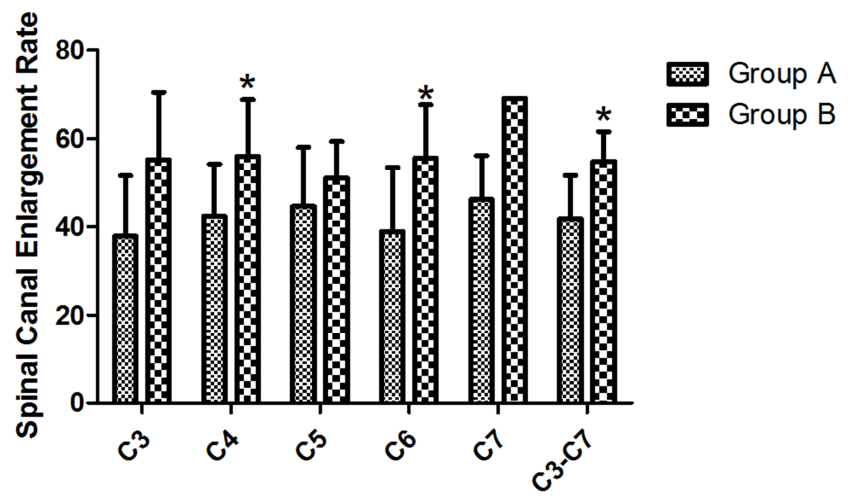

Figure 2. Comparison of spinal canal enlargement rate (\%) of each segment between the two groups.

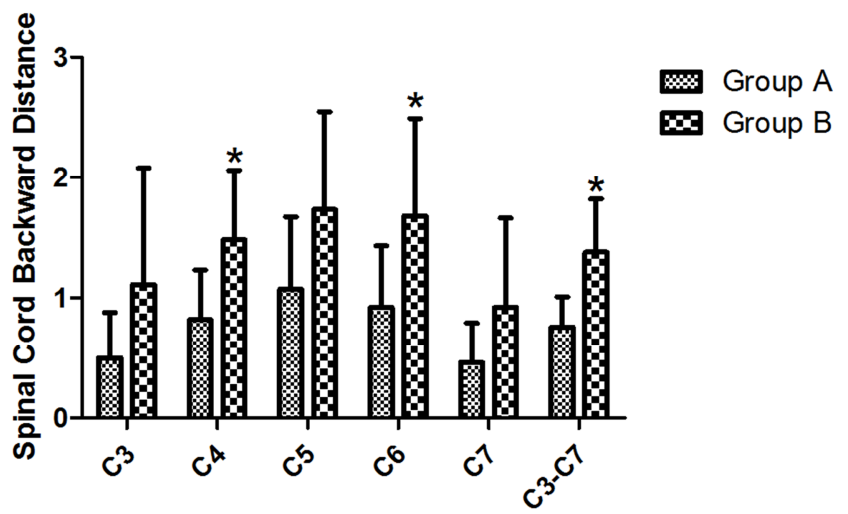

Figure 3. Comparison of the distance $(\mathrm{mm})$ of each segment of the spinal cord between the two groups.

\section{Discussion}

Cervical spondylotic myelopathy is due to the degeneration of the cervical spine compressing the cervical spinal cord [10]. For patients with significant cervical spinal cord compression, surgery is recommended as soon as possible. Centerpiece micro titanium plate is a new type of internal fixation material used to fix the lamina opened during single cervical spine surgery in recent years [11]. It is a simple, safe and effective method for the treatment of cervical spondylotic myelopathy. However, there are a series of complications after posterior cervical single-door spinal canalplasty, such as axial symptoms and 5 cervical nerve root palsy, decreased cervical spine mobility, straightening of cervical spine physiological curvature or even kyphosis.

Cervical 5 nerve root palsy after cervical spine decompression is the new occurrence of deltoid and/or biceps palsy when the original spinal cord symptoms do not worsen after cervical spine decompression. Most patients only show mild muscle strength decrease, but a small number of patients may be associated with sensory disturbance. Stoops et al. first reported cervical 5 nerve root palsy after cervical laminectomy in 1961 [12]. Cervical 5 nerve root palsy after posterior cervical decompression is mainly manifested as weakness of one or both deltoid muscles or biceps brachii muscles, skin sensory disturbances in the area innervated by C5 nerves. Most patients have the above symptoms within 1 week after surgery, and a small number of patients can appear within 2 to 4 weeks after surgery [13]. In this study, patients in group B had cervical 5 nerve root palsy after 2 days to 1 week, which was manifested as a decrease in biceps or deltoid muscle strength compared with before operation.

At present, there is no specific and recognized complete program for the prevention and treatment of C5 nerve root palsy. However, scholars have proposed many preventive measures, including intraoperative neuroelectrophysiological testing [14], preventive foraminal expansion and decompression [15], dural incision and some non-surgical preventive measures. Treatment measures include hormone combined with dehydration therapy, hyperbaric oxygen or low-frequency wave therapy, rest and muscle strengthening exercises. In this study, the incidence of cervical 5 nerve root palsy was $12.90 \%$, which is similar to the reported rate. In addition, the average spinal canal enlargement rate and the distance of the spinal cord in group B is more than group A, indicating that the occurrence of postoperative $\mathrm{C} 5$ nerve root palsy is more than the extent of spinal canal enlargement and the distance of spinal cord. Furthermore, our results showed that the occurrence of postoperative $\mathrm{C} 5$ nerve root palsy is related to the lamina door opening angle, especially $\mathrm{C} 4 \sim \mathrm{C} 6$ lamina door opening angle.

\section{Conclusion}

In conclusion, our results found that controlling the opening angle of the lamina, the expansion rate of the spinal canal, and the distance of the spinal cord may prevent the occurrence of cervical 5 nerve root palsy in cervical expansive open-door laminoplasty.

\section{References}

[1] Bakhsheshian J, Mehta VA, Liu JC: Current Diagnosis and Management of Cervical Spondylotic Myelopathy. Global Spine J 2017, 7 (6): 572-586. 
[2] Fan XW, Wang ZW, Gao XD, Ding WY, Yang DL: The change of cervical sagittal parameters plays an important role in clinical outcomes of cervical spondylotic myelopathy after multi-level anterior cervical discectomy and fusion. J Orthop Surg Res 2019, 14 (1): 429.

[3] Abiola R, Rubery P, Mesfin A: Ossification of the Posterior Longitudinal Ligament: Etiology, Diagnosis, and Outcomes of Nonoperative and Operative Management. Global Spine J 2016, 6 (2): 195-204.

[4] Hirabayashi K, Satomi K: Operative procedure and results of expansive open-door laminoplasty. Spine (Phila $\mathrm{Pa} 1976)$ 1988, 13 (7): 870-876.

[5] Li N, Tian W, Yuan Q, He D: Cervical Spondylotic Myelopathy due to the Ochronotic Arthropathy of the Cervical Spine. J Korean Neurosurg S 2016, 59 (1): 65-68.

[6] Shang ZK, Zhang YZ, Zhang D, Ding WY, Shen Y: Clinical and Radiological Analysis of Bryan Cervical Artificial Disc Replacement for "Skip" Multi-Segment Cervical Spondylosis: Long-Term Follow-Up Results. Med Sci Monitor 2017, 23: 5254-5263.

[7] Lim CH, Roh SW, Rhim SC, Jeon SR: Clinical analysis of C5 palsy after cervical decompression surgery: relationship between recovery duration and clinical and radiological factors. Eur Spine J 2017, 26 (4): 1101-1110.

[8] Xu XN, Liu HY: [Clinical analysis of cervical posterior internal fixation titanium plate and silk suspension internal fixation cervical spondylosis]. Zhonghua Wai Ke Za Zhi 2017, 55 (3): 214-219.
[9] Lee JY, Sharan A, Baron EM, Lim MR, Grossman E, Albert TJ, Vaccaro AR, Hilibrand AS: Quantitative prediction of spinal cord drift after cervical laminectomy and arthrodesis. Spine (Phila Pa 1976) 2006, 31 (16): 1795-1798.

[10] Wu W, Yang Z, Zhang T, Ru N, Zhang F, Wu B, Liang J: Microstructural Changes in Compressed Cervical Spinal Cord Are Consistent With Clinical Symptoms and Symptom Duration. Spine (Phila Pa 1976) 2020, 45 (16): E999-E1005.

[11] Liu FY, Ma L, Huo LS, Cao YX, Yang DL, Wang H, Yang SD, Ding WY: Mini-plate fixation versus suture suspensory fixation in cervical laminoplasty: A meta-analysis. Medicine (Baltimore) 2017, 96 (5): e6026.

[12] Stoops WL, King RB: Neural complications of cervical spondylosis: their response to laminectomy and foramenotomy. J Neurosurg 1962, 19: 986-999.

[13] Sakaura H, Hosono N, Mukai Y, Ishii T, Yoshikawa H: C5 palsy after decompression surgery for cervical myelopathy: review of the literature. Spine (Phila Pa 1976) 2003, 28 (21): 2447-2451.

[14] Fan D, Schwartz DM, Vaccaro AR, Hilibrand AS, Albert TJ: Intraoperative neurophysiologic detection of iatrogenic C5 nerve root injury during laminectomy for cervical compression myelopathy. Spine (Phila Pa 1976) 2002, 27 (22): 2499-2502.

[15] Steinmetz MP, Resnick DK: Cervical laminoplasty. Spine J 2006, 6 (6 Suppl): 274S-281S. 